\title{
14. WHITE DWARF ATMOSPHERES
}

\author{
V. WEIDEMANN \\ Institut für Theoretische Physik und Sternwarte der Universität, Kiel, Germany
}

\section{Introduction, Surface Gravity}

We first consider the general information scheme for the interpretation of observational data (Figure 1). From the relations plotted it is evident that (in going from left to right) this scheme can only be solved if distances are known and if we are able to determine the atmospheric parameters: effective temperature, $T_{\text {eff }}$, surface gravity, $g$, and chemical composition from observations of colors and spectra - which is the genuine task of the theory of stellar atmospheres.

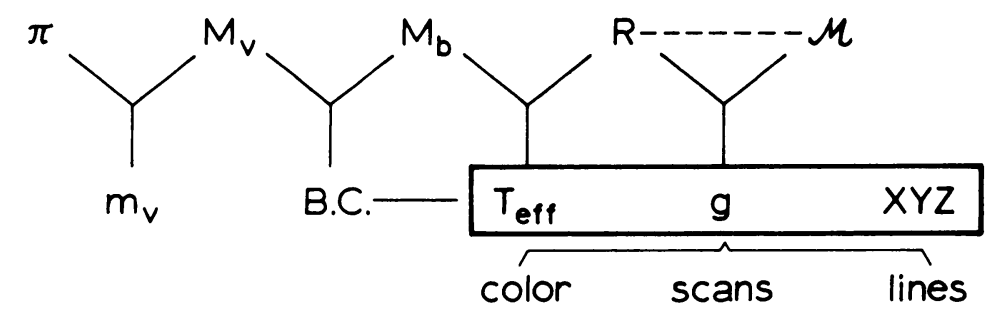

$$
\begin{aligned}
& \log R / R_{\odot}=-2 \log T_{\text {eff }}-0.2\left(M_{v}+\text { B.C. }\right)+8.45 \\
& \log \mathscr{H} / \mathscr{M}_{\odot}=-2 \log R / R_{\odot}+\log \mathrm{g}-4.44
\end{aligned}
$$

Fig. 1. Information scheme displaying relations between observed and derived quantities: $\pi$ : parallax, $m_{v}$ and $M_{v}$ : apparent and absolute visual magnitude, $M_{b}$ : bolometric magnitude, B.C.: bolometric correction, $R$ and $\mathscr{M}$ : radius and mass (broken line indicates mass-radius relation), the box contains the atmospheric parameters: effective temperature, surface gravity $g$ and chemical composition $X Y Z$.

A possibility to solve the scheme in a simpler way is given in the special case only where in addition the mass is known: in this case the problem reduces to a determination of $T_{\text {eff }}$ (and bolometric correction B.C.) and $g$ is a derived quantity. If on the other hand a mass-radius relation for degenerate configurations is assumed to hold, (or if reliable gravitational redshifts were available) the scheme can completely be solved in going from right to left, as soon as $g$ and $T_{\text {eff }}$ are determined. With this scheme in mind it is easy to bring order into the history of white dwarfs investigations. I shall not do this here but just demonstrate how the surface gravity in a typical white dwarf atmosphere can be deduced in the best observed case of 40 Eri B $\left(\mathscr{M}=(0.43 \pm 0.04) \mathscr{M}_{\odot}, M_{v}=11.0\right)$. With a very rough estimate of $T_{\text {eff }}, 10000<T_{\text {eff }}<$ $<20000 \mathrm{~K}\left(\Delta \log T_{\text {eff }}= \pm 0.15\right)$, B.C. $=(1.0 \pm 0.7)$ we obtain $\log R / R_{\odot}=-1.85 \pm 0.45$, and, finally $\log g=7.7 \pm 0.9$. 
Since this combination of mass and radius fulfills the Chandrasekhar relation for completely degenerate configurations (with $\mu_{e}=2$ ) very well, and since 40 Eri $\mathrm{B}$ is a typical white dwart of the most common spectral type DA we understand that many have taken the validity of the $\mathscr{M}-R$ relation for granted and sometimes used it in an uncritical way. As demonstrated by the example of 40 Eri B the $g$-determination can be improved if a temperature scale is established. Greenstein's first attempt (1958) was based on calculations of $U-V$ colors with gray atmospheres and estimates of the influence of the Balmer jump on the $U$ band. For 22 white dwarfs with known distances he found the radii to scatter about a factor 2 only around a mean value of $\log R / R_{\odot}=$ -1.90 . With the $\mathscr{M}-R$ relation this would imply a range on $\log g$ from about 7 to 8.7. Independent information about the surface gravity can be obtained from line broadening theory, a method which was applied extensively by Weidemann in the case of van Maanen 2 (1960) and the DA white dwarfs (1963) to give $\log g=8 \pm 0.5$, with a resulting large scatter around the mass-radius relation in those cases where distances were known. In view of the uncertainties in parallaxes and atmospheric parameter determinations it was then only stated that nothing seemed to be in contradiction with a mass-radius relation of $\mu_{e}=2$. While this point will be taken up later again, we stress at the moment the fact that the surface gravity in white dwarf atmospheres is 1000 to 10000 times higher than in main sequence stars.

\section{DA Atmospheres}

For a first estimate on the physical conditions in such high gravity atmospheres with a normal hydrogen-rich composition we can go back to the work of Vitense (1951) who calculated absorption coefficients and the dependence of gas and electron pressure, Balmer jump and other spectral characteristics on effective temperature and gravity all the way up to $\log g=8$ (see also Unsöld, 1955). The main result is that the high gravity compresses the atmospheres such as to increase the gas pressure by a factor of about 100 as compared to main sequence stars. A cor respondingly higher electron pressure is not only responsible for the extreme broadening of the Balmer lines (which overlap and disappear beyond $\mathrm{H}_{\zeta}$ ) but also for a generally lower ionization degree which shifts the influence of $\mathrm{H}^{-}$to higher temperatures and causes Balmer jump and lines to weaken below $T_{\text {eff }} \approx 12000 \mathrm{~K}$. We thus understand Figure 2 (Unsöld, 1955; Weidemann, 1966) which is indispensable for a qualitative explanation of the DA distribution in the two-color diagram. Figure 3 summarizes schematically what has been discussed in more detail elsewhere (Weidemann, 1963, 1966): its essence is to consider deviations from a black-body flux distribution (Balmer jump, Balmer lines, $\mathrm{H}^{-}$maximum) as being reflected in corresponding deviations of the UBV position from the UBV black-body line. Each position is reached in a sequence of steps: blackbody to line-free model, (i.e. Balmer jump, $\mathrm{H}^{-}$shift of the flux to the blue and violet) line-free model to blanketed model (i.e. influence mainly of lines on the stratification and therefore the continuous flux distribution, f.e. by backwarming) and finally corrections for line-blocking effects in the filter bands. 


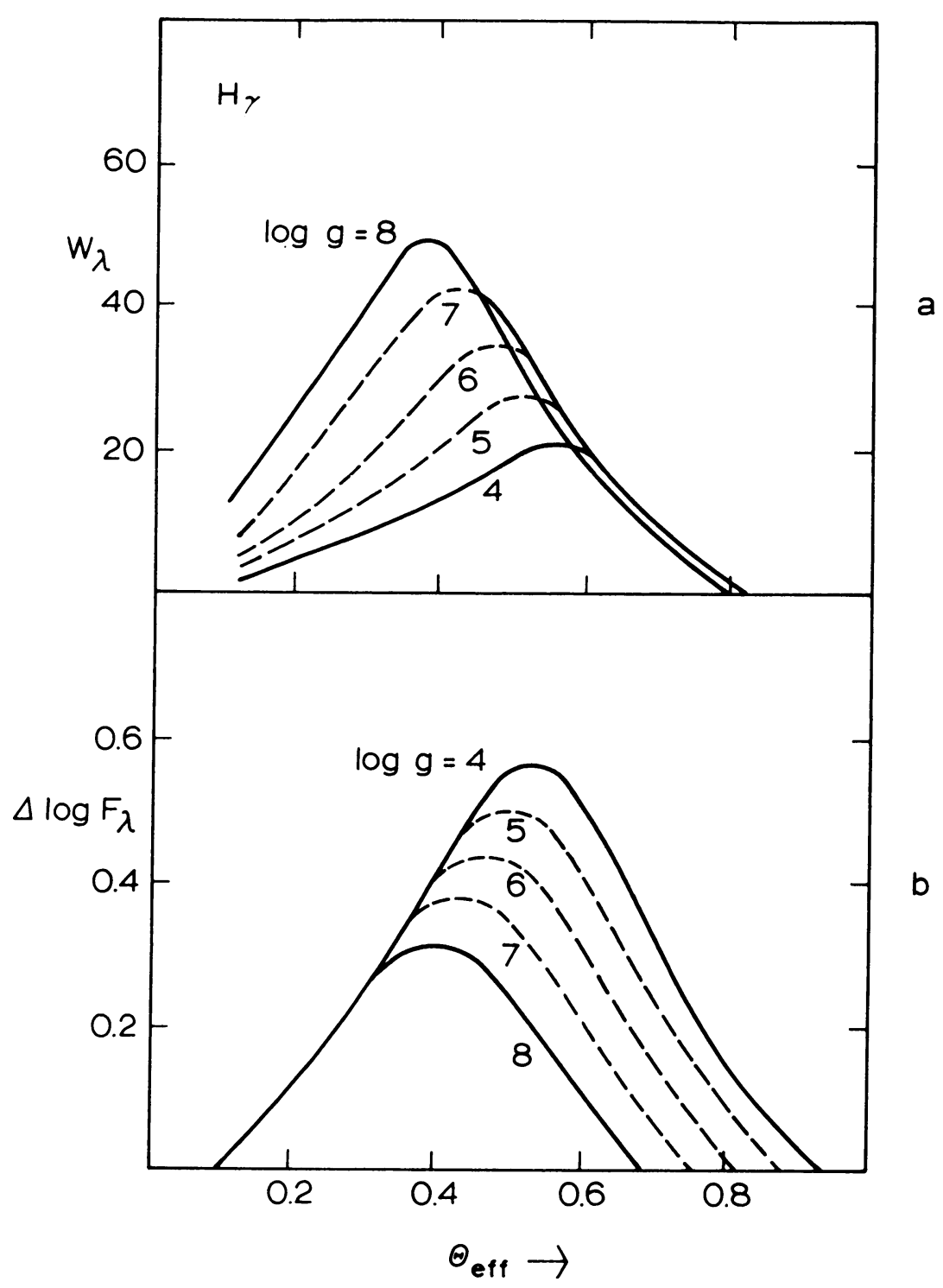

Figs. 2a, 2b. (a) Equivalent width of $\mathrm{H} \gamma$ and (b) Balmer jump $\Delta \log F_{\lambda}$ as a function of $\Theta_{\text {eff }}=$ $5040 / T_{\text {eff }}$, with $\log g$ as parameter. With increasing $g$ the maxima are shifted towards higher $T_{\text {eff }}$ due to increasing $\mathrm{H}^{-}$absorption.

These results were quantitatively confirmed by the model calculations of Matsushima and Terashita (1969a), Terashita and Matsushima $(1966,1969)$ and can be used as an independent method of gravity determination from UBV data (Figure 4) again confirming $\log g \approx 8$ (details will be discussed later). New information is available from intermediate-band photometry by Graham (1970). From Figures 4 and 5 which show essentially line-free positions we may draw two conclusions: 
(a) The fact that the DA white dwarfs lie close to the blackbody line in the Johnson system two-color diagram is caused by line-blocking effects rather than a smoothing of the continuum.

(b) The black-body line is not a natural limit for white dwarfs - if there were white

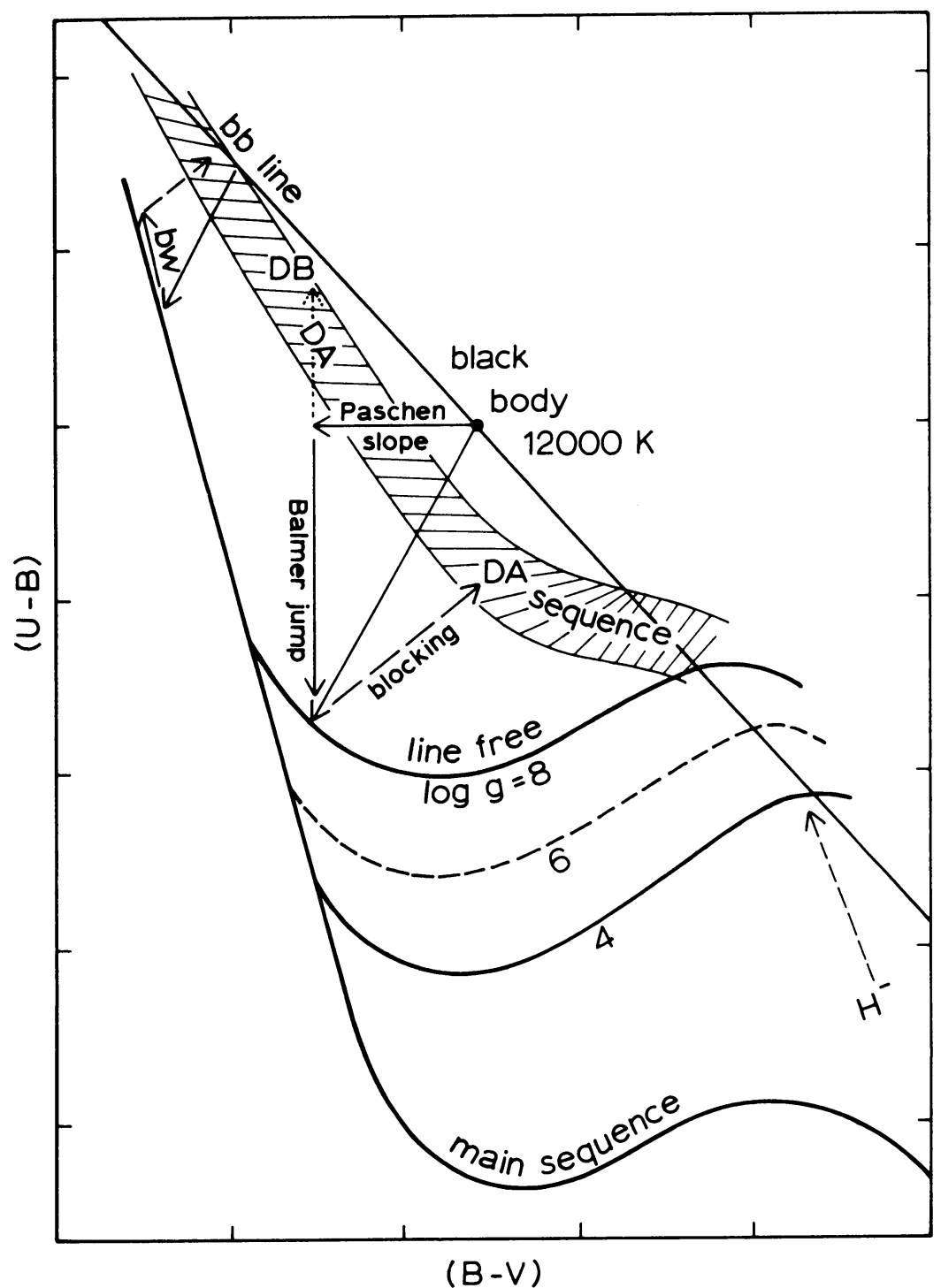

Fig. 3. Schematical behavior of DA white dwarfs in the UBV diagram: the deviations from a black-body flux-distribution (Paschen slope, Balmer jump) add up to give the line-free positions, from which a blocking vector (broken line) reaches the observed position. Upper left: a backwarming vector (bw) operates along the line-free position curve and causes the star to appear hotter. Lower right: $\mathrm{H}^{-}$-absorption, strongest in the red, shifts the flux to the blue and the ultraviolet (broken arrow). Dotted arrow points to DB position (see text on p. 94). 


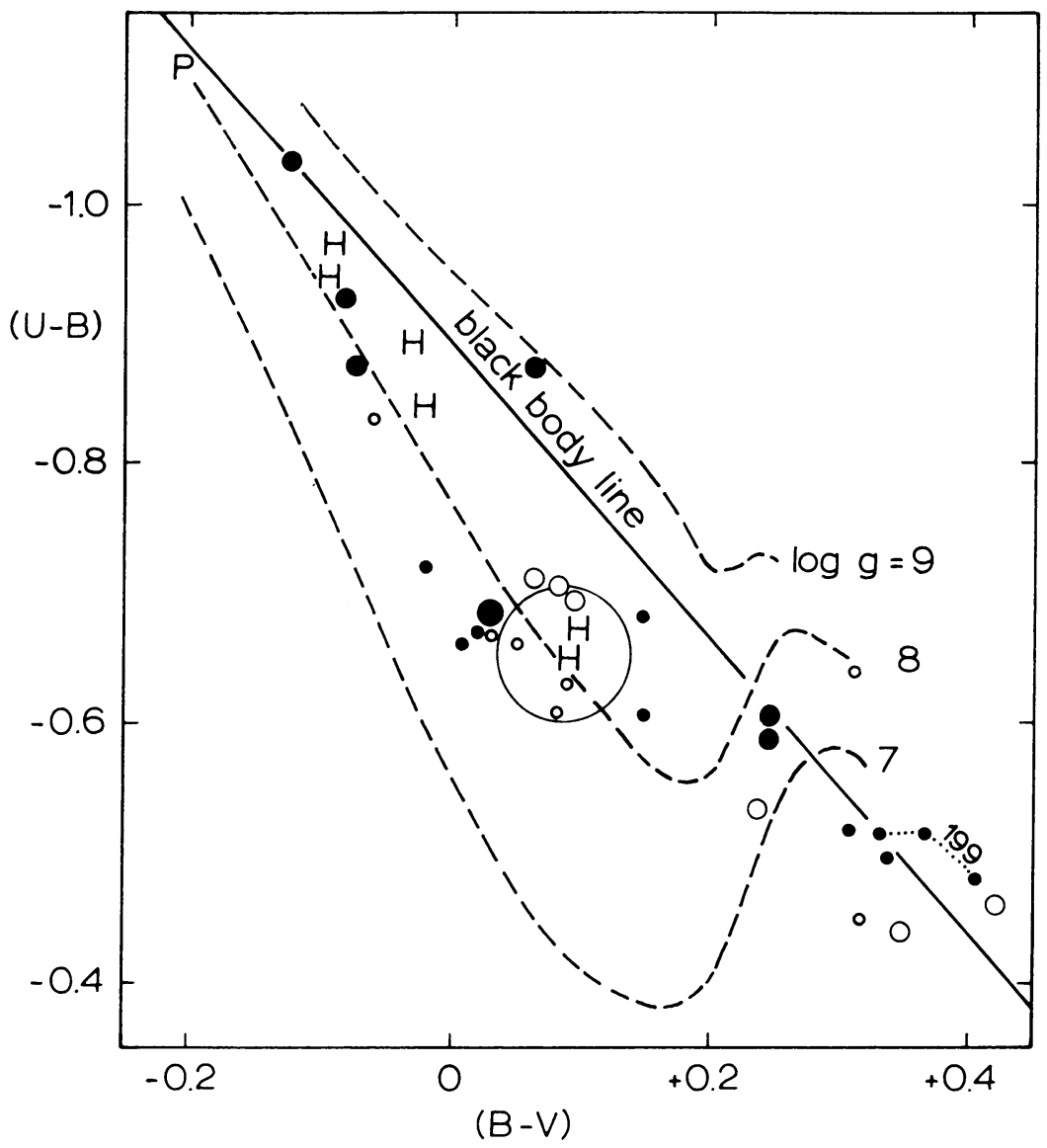

Fig. 4. UBV diagram with black-body line, theoretical positions for $\log g=7,8$ and 9 , according to Terashita and Matsushima (1969) (broken lines), and observed DA white dwarfs with known distances (Eggen, 1969): $\bigcirc 40$ Eri B, ○: Eggen Table I, $\bigcirc$ : Table II, •: Table IV, ○: Table VI, H: Hyades, P: Pleiades. Dotted line connects different positions for EG 199. Circle indicates maximum error of $0.05 \mathrm{mag}$.

dwarfs with $\log g$ about 9 , we would according to Figure 4 expect them above this line (where e.g. many blue high galactic latitude objects are found).

In a similar way the behavior of the Balmer lines can be understood. Whereas the equivalent width reaches a maximum at $T_{\text {eff }}=12000 \mathrm{~K}$ for $\log g=8$ (Figure 2) due to the increased $\mathrm{H}^{-}$opacity, the central intensity decreases all the way from the hottest to the coolest DA stars. At the cool end of the sequence the Balmer lines thus appear sharp (DAs, DAss), at the hotter shallow (DAwk). The variation of the profile shape of $\mathrm{H} \gamma$ as a function of both $T_{\text {eff }}$ and $g$ has been determined first semi-empirically by Weidemann (1963), then calculated by Matsushima and Terashita (1966), and with a revised Stark broadening theory by Terashita and Matsushima (1969). Whereas I used these profiles in 1963 to derive (essentially relative) $T_{\text {eff }}$ and $g$-values for 22 DA 


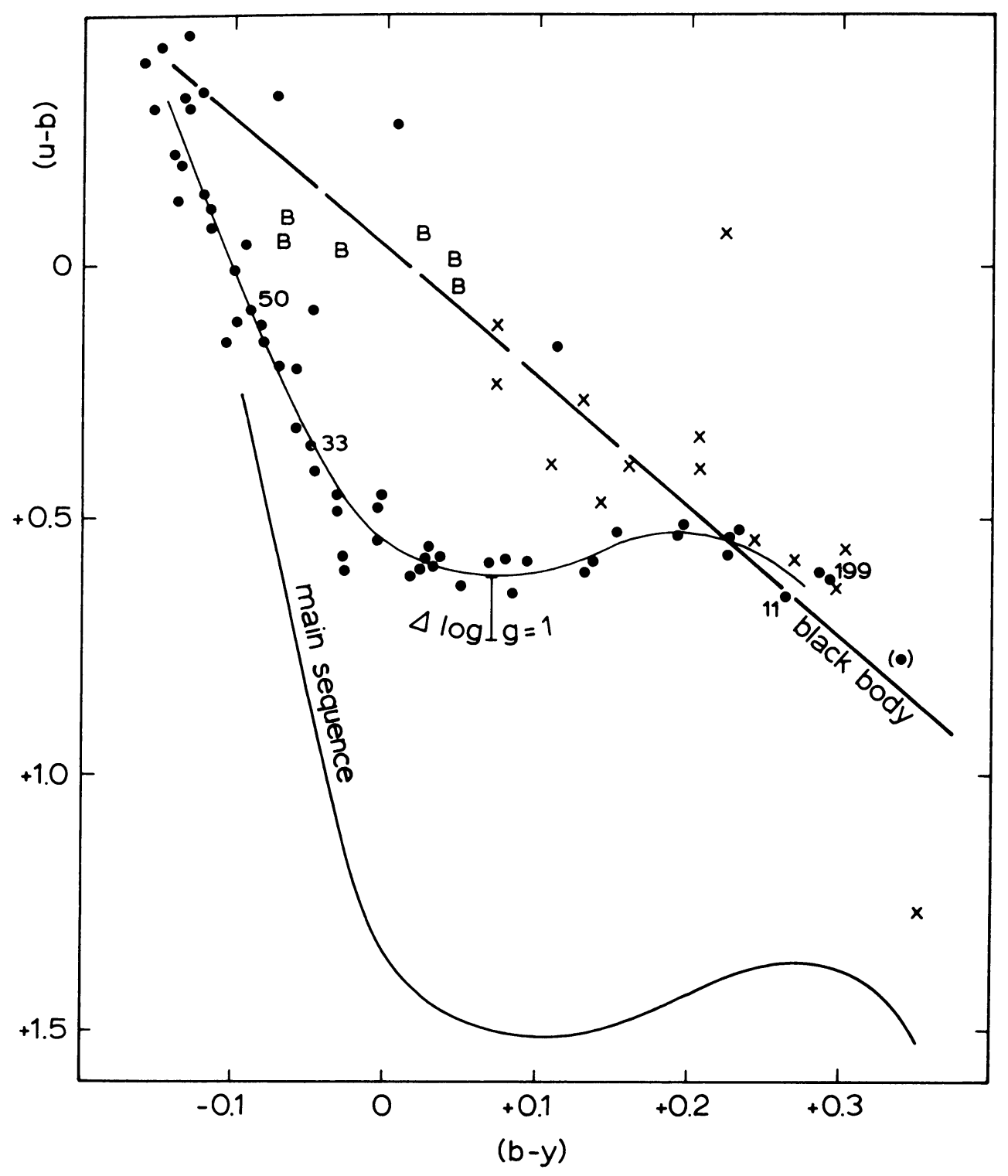

Fig. 5. Two-color diagram in the Strömgren system, according to Graham (1970). Dots: DA (and DO) white dwarfs, B: DB, crosses: other non-DA white dwarfs (O): EG 87, composite, not reliable. Mean DA sequence outlined. Bar indicates theoretical shift for a gravity change (cf. Figure 3 and text, p. 88).

stars, Matsushima and Terashita preferred to determine these parameters from the two-color diagram (Figure 4) since their (purely theoretical) model-atmosphere approach did not reproduce the observed profiles well enough. Considerable differences in gravity determinations between Matsushima and Terashita (1966) and 
Terashita and Matsushima (1969) are due to the fact that different line-broadening theories have been used. Since the broadening theory is still under discussion, it seems that even now line-profile data should be used only differentially for the determination of atmospheric parameters.

As far as the models themselves are concerned, the full capacity of computational facilities has been applied in the second half of the past decade by Matsushima and
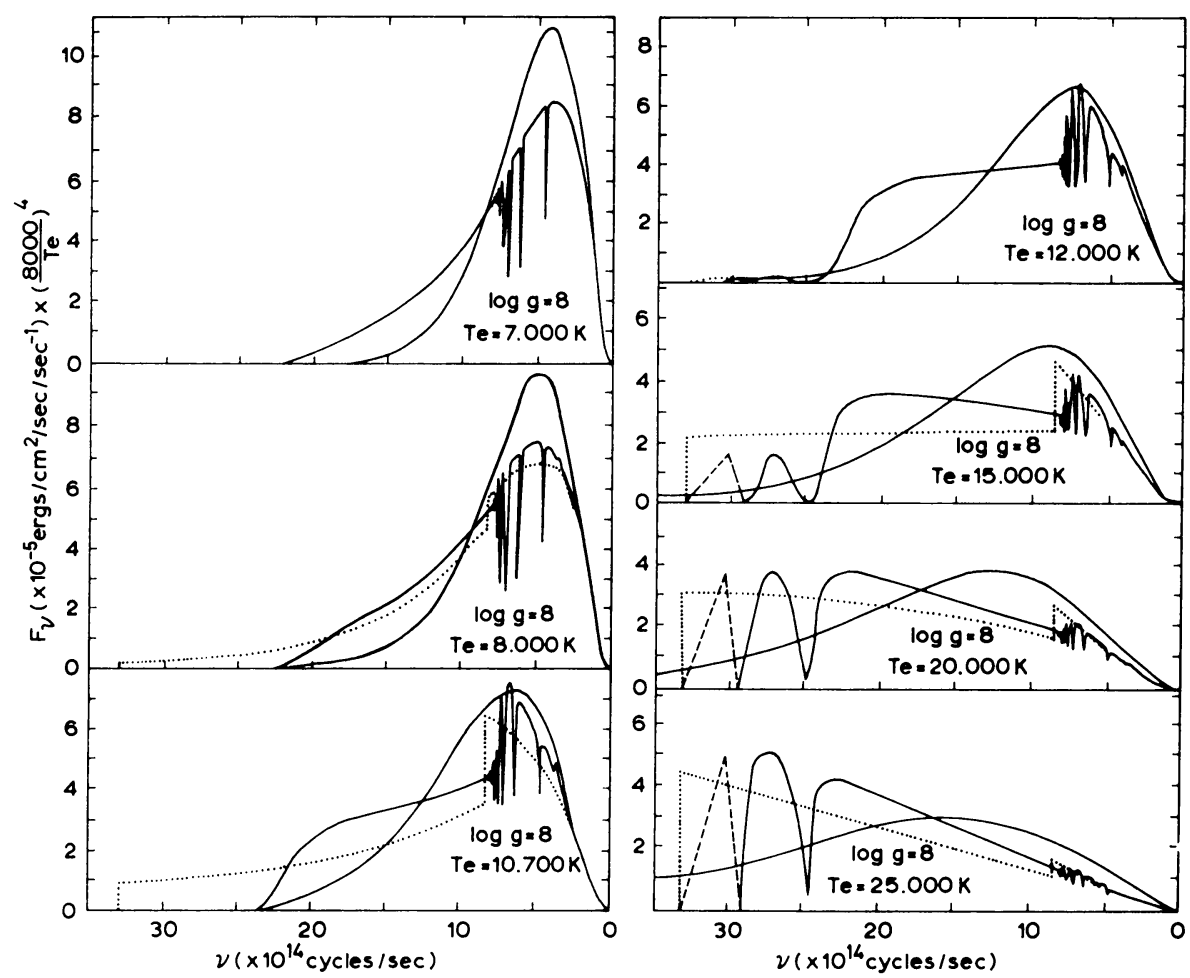

Fig. 6. Flux distributions for DA model-atmospheres, $\log g=8$, according to Terashita and Matsushima (1969). Dotted: non-blanketed models. Black-body distribution for comparison.

Terashita. Although the first approach (1966) in which radiative flux-constant unblanketed models were calculated was only partly successful (Weidemann, 1968), the newer models in which the blanketing by hydrogen lines was fully taken into account (Terashita and Matsushima, 1969) should constitute as real step ahead. Figure 6(reproduced from TM, 1969) clearly demonstrates how Lyman absorption causes backwarming (especially for the hotter models) and how the emergent flux in the visual regions changes. For the first time bolometric corrections were derived and a new temperature scale was established. Terashita and Matsushima were thus able to solve our information scheme (Figure 1) with higher accuracy than before and determined radii and 
masses for 30 DA stars with known luminosities. They finally went on (MT, 1969b) to admit variations in the ratio of hydrogen to helium abundances, and considered the special case of 40 Eri B (MT, 1969c). With all other data known a value of 0.90 for the fraction of hydrogen by number was derived. Although the absolute data given should not be considered final in view of the fact that metals were not included as opacity sources (see below, p. 92), and in view of discrepancies to be discussed later these papers demonstrate clearly the power of computer methods in the case of white dwarf atmospheres. However, doubts arise if we next turn to the question of the significance of the results. Radii and masses as determined by Terashita and Matsushima

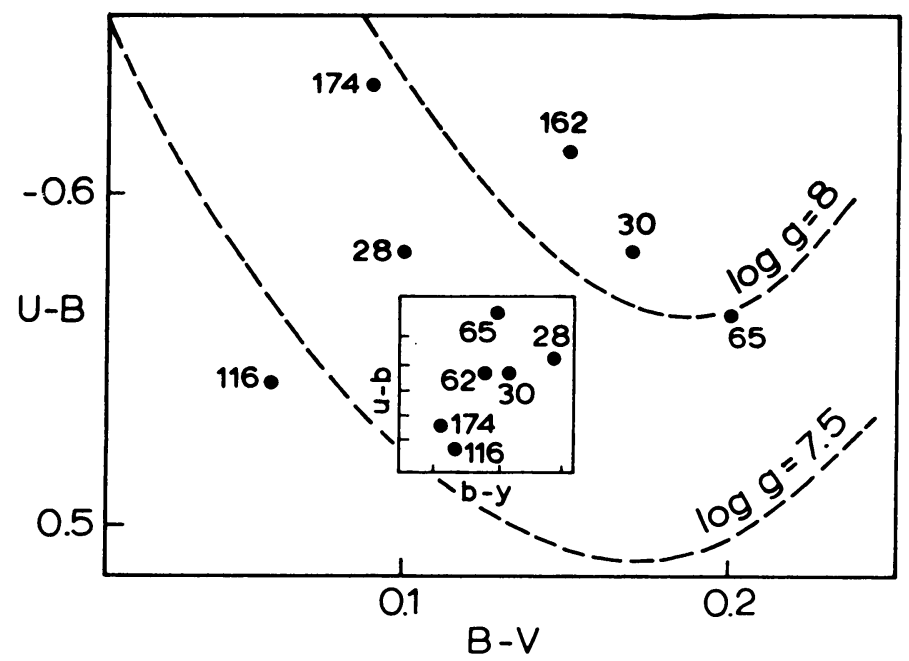

Fig. 7. Comparison of UBV and uby data for six DA stars. Broken lines in the UBV diagram indicate theoretical lines for constant $g$ according to Terashita and Matsushima (1969). Insert is adjusted in scale such as to cover corresponding intervals in $(u-b)$ (scale $0.01 \mathrm{mag})$ and $(b-y)$ (scale $0.01 \mathrm{mag})$. Graham error (1969) is $\pm 0.01 \mathrm{mag}$ in $(\mathrm{b}-\mathrm{y})$ and $\pm 0.015 \mathrm{in}(\mathrm{u}-\mathrm{b})$.

(1969) do show a very large scatter around the theoretical mass-radius relation (Figure 9 ). Since the radii were derived from absolute visual magnitudes via $T_{\text {eff }}$ and masses from radii with $\log g$, and since both, $T_{\text {eff }}$ and $\log g$ were read out from the position of individual stars in the two-color diagram (Figure 4) the reliability of the results in this approach depends on both the significance of the scatter in the color-magnitude diagram and the UBV diagram.

In this respect the narrowness of the DA sequence in Graham's two-color diagram (Figure 5) teaches us that most of the scatter in Figure 4 cannot be significant. This was evident in some cases for which UBV has been determined repeatedly - e.g. for LP 9-231 (EG 199) (van Altena, 1966; Luyten, 1967; Eggen and Greenstein, 1967; Eggen, 1969) differences up to $0.08 \mathrm{mag}$ in $(\mathrm{B}-\mathrm{V})$ or $0.12 \mathrm{mag}$ in $(\mathrm{U}-\mathrm{V})$ occur in the published data. But more convincing is the following consideration. We first extract 
from the predicted locations for line-free flux distributions (Figure 3) that the $g$ dependence in the Strömgren system is largest for the cooler DA stars with $0<(b-y)$ $<+0.2$. From Figure 3 and Figure $5-$ in which a bar indicates the shift of the linefree sequence for $\Delta \log g=-1$, calculated with TM fluxes (1969) - we then see that this part of the Graham sequence implies for the observed DA stars $\log g=$ const. to within \pm 0.15 . If we furthermore check if there is any correlation between relative positions in the Johnson and the Strömgren two-color diagrams we obtain a negative result. Figure 7 enlarges a part of both diagrams in comparable scales and shows how a group of 6 DA white dwarfs which in the Strömgren system have nearly identical positions are 'blown up' in the Johnson system. Again corrections up to 0.08 mag in UBV colors must be invoked in order to obtain a comparable scatter in $\log g$.

Finally I plotted a histogram (Figure 8) which shows how the scatter in $g$-values derived is reduced by changing from Johnson to Strömgren colors. The obvious conclusion is that the DA stars do in reality form a rather narrow sequence and that the $g$-scatter derived from the UBV diagram is not significant. It follows that the DA

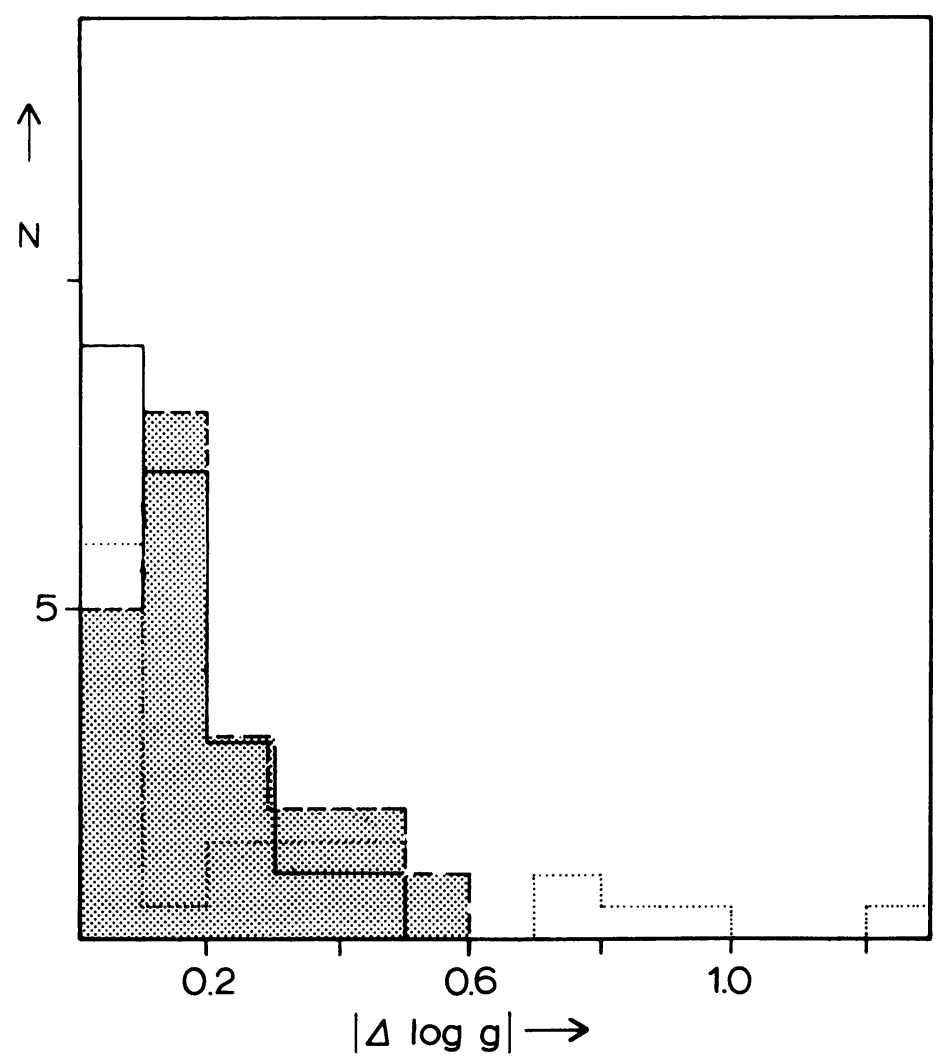

Fig. 8. Distribution of DA white dwarfs around mean sequence of constant gravity. Full line: on using data from Graham's two-color diagram (Strömgren system) (1970); dashed line, shaded area: from UBV diagram (Figure 4); dotted: from $M_{v}-(\mathrm{U}-\mathrm{V})$ diagram (Figure 10). 


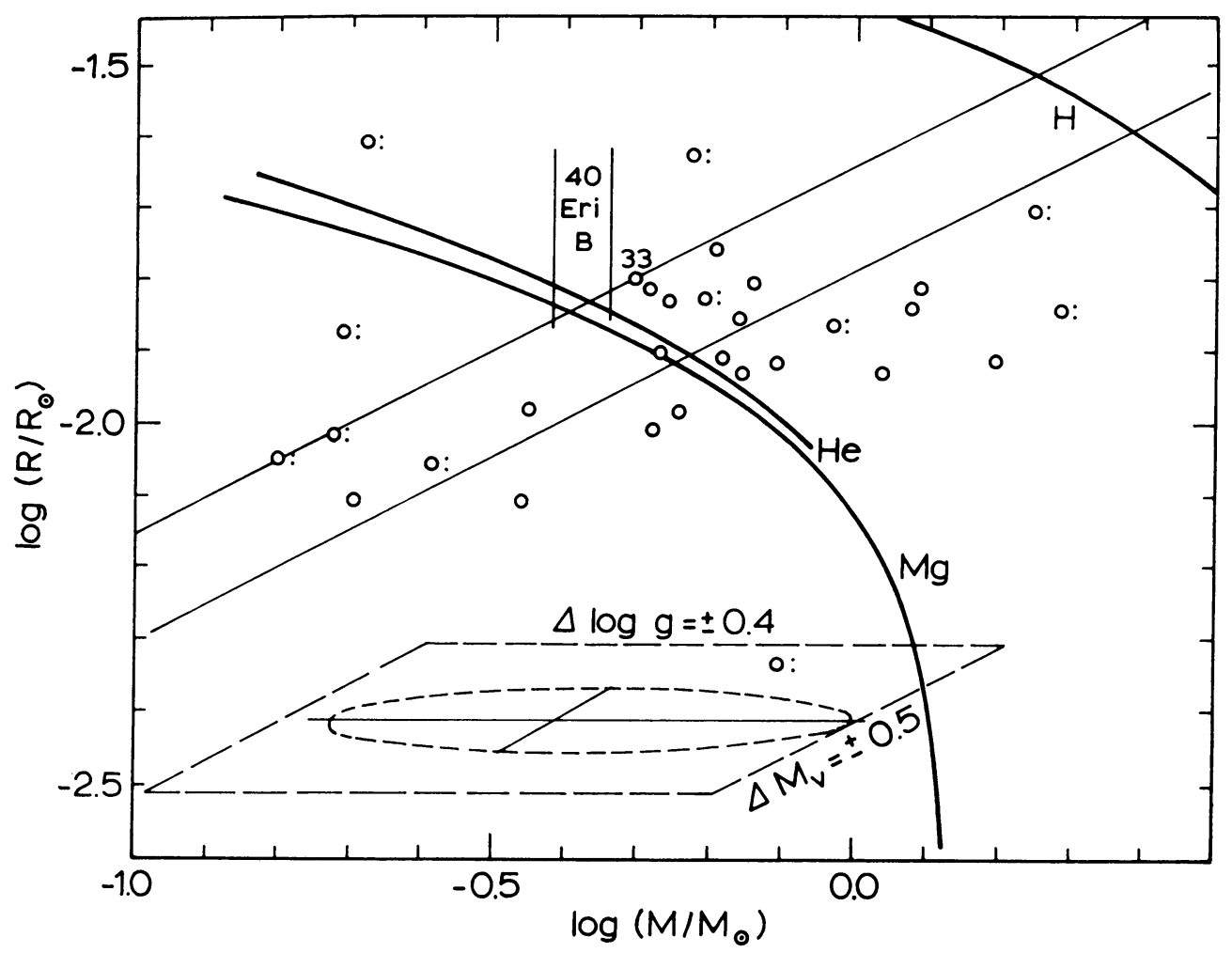

Fig. 9. Mass-radius diagram, as given by Terashita and Matsushima (1969) with additions: diagonal strip indicating $\log g=$ const. \pm 0.15 , to which white dwarfs should be confined if Graham's data and interpretation given here are accepted; vertical strip: mass range of 40 Eri B (EG 33); lower part insert: error ellipse corresponding to error circle in UBV-diagram of Figure 4, dashed lines indicating total error area including luminosity error. Notice same general size and direction of derived white dwarf data cloud. Colons mark stars for which either distance or spectral type is called uncertain in the literature.

stars in the mass-radius diagram of Terashita and Matsushima (1969) should be confined to a narrow strip around $\log g=$ const. (Figure 9). The remaining scatter is then entirely due to scatter in radii. From the shape of the distribution alone it can be concluded that this scatter too has probably no physical meaning since if real it would imply a mass-radius relation which runs just diagonal to the Chandrasekhar relation for degenerate stars. Although it cannot be excluded that there are some objects with extended hydrogen shells and corresponding larger radii (Hamada and Salpeter, 1961) it is more probable that the entire scatter is due to errors in UBV colors and distance determinations. (See error area outlined in Figure 9.) In order to check this further we consider finally the color-magnitude diagram (Figure 10). It shows that the stars with better parallaxes (large dots, Eggen, 1969 and 1970) do not contradict a theoretical mean relation for completely degenerate configurations of $\log g=$ const. (broken lines, according to Matsushima and Terashita, 1969a). [In the case of 


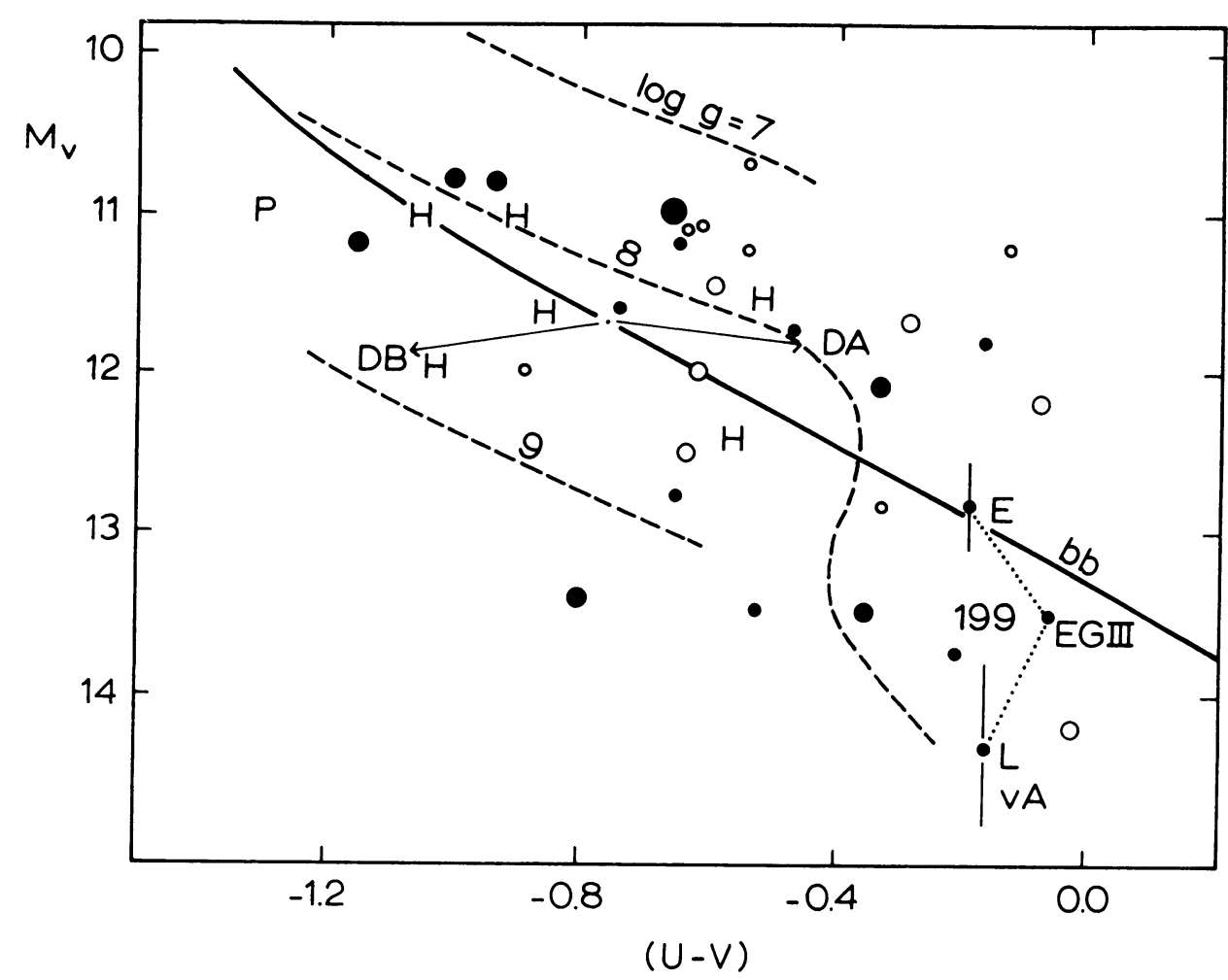

Fig. 10. Color-magnitude diagram for DA stars. Symbols as explained in legend to Figure 4. Dashed lines: theoretical lines for constant radii (and corresponding gravities for fully degenerate configurations) according to Matsushima and Terashita (1969a) with black-body line for $\log g=8$, $\log R / R_{\odot}=-1.91$, added. Arrows arising from black body at $12000 \mathrm{~K}$ demonstrate differences of theoretical DA and DB positions at equal $T_{\text {eff }}$ (cf. Figure 3 and text on p. 94).

Lower right: different data extracted from the literature for EG 199: EG III (Eggen and Greenstein, 1967), L (Luyten, 1967), vA (van Altena, 1966), E (Eggen, 1969) indicate extreme uncertainties of $M_{r}$ determinations in some cases.

EG 165, far to the left, $U-V$ is probably in error since it fits the sequence of the other objects in the $M_{I}(\mathrm{R}-\mathrm{I})$ diagram of Eggen (1970)]. The large discrepancies of individual parallax determinations are demonstrated in the (admittedly extreme) case of EG 199.

Correspondingly the scatter of $\log g$ if derived from this diagram via the $M-R$ relation would be largest (see histogram Figure 8, dotted line). We thus conclude that there is strong evidence that the DA white dwarfs are confined to essentially constant surface gravity and do obey the mass-radius relation. The absolute figures depend somewhat on the scale calibrations: my best estimate is at present $\log g=$ $7.90 \pm 0.15$ corresponding to masses between 0.42 and 0.60 solar masses. In view of the importance of this conclusion we must now ask how far it depends on uncertainties or imperfections of atmospheric theory. A few facts must be mentioned in this connection 
(1) From a comparison between the shape of the theoretical curves for $\log g=$ const. in both two-color diagrams and the observed DA distributions in Figure 4 and Figure 5 it is evident that Terashita and Matsushima's calculations predict a too pronounced S-shape at the cool end of the DA sequence. This may also be concluded by a comparison of the theoretical color-magnitude relation (Figure 10) with the observed monotonic decrease of $\mathrm{W}_{\lambda}(\mathrm{H} \gamma)$ as a function of $(\mathrm{U}-\mathrm{V})$ (see e.g. Greenstein, Figure 3 , 1969). Since the turn-up of the DA sequence at the cool end is caused by $\mathrm{H}^{-}$ (Figure 3) one probably has to invoke a reduction of $\mathrm{H}^{-}$relatively to other opacity sources. At lower temperature the atmospheres become extremely neutral with $\log \left(P_{g} / P_{e}\right) \approx 4$. Metals and molecule formation cannot be neglected and may be responsible for the discrepancies mentioned. Unfortunately we have no observational hint as yet on metal abundances in DA stars. Assuming it to be normal we do expect extreme blanketing and blocking effect on coming down to solar temperature (see Weidemann, 1966). A study is underway at Kiel to get some quantitative results.

(2) As for the reliability of the TM temperature scale at higher temperatures, around $15000 \mathrm{~K}$, doubts remain since backwarming effects caused by metal absorption in the UV should become important. For main sequence stars, Davis and Webb (1970) have pointed out that Mihalas hydrogen-line-blanketed models - which correspond in the degree of sophistication to those of Terashita and Matsushima - yield upper limits to effective temperatures only, since the space-observed UV depressions are larger, whereas Adams and Morton (1968) have shown that metal line blanketing causes backwarming amounting to about 1000 degrees at $T_{\text {eff }}$ around $16000 \mathrm{~K}$.

For white dwarfs with normal metal abundances UV line blanketing should be even stronger due to increased pressure broadening.

Indeed, Graham's most accurate data (1970) give some indication that the temperature scale should be stretched between 40 Eri B (EG 33) - for which Matsushima and Terashita, 1969c, give $T_{\text {eff }}=(15300 \pm 380) \mathrm{K}-$ and He 3 (EG 50), : a dip in the $m_{1}$ vs. $\mathrm{b}-\mathrm{y}$, correspondingly stronger in the $c_{1}$ vs. $\mathrm{u}-\mathrm{y}$ diagram and a plateau between both stars in both the $M_{v}$ vs. $\mathrm{u}-\mathrm{y}$ and $\mathrm{U}-\mathrm{V}$ color magnitude diagrams point all into this direction. The sudden steepening of the visual flux distribution necessary to explain these phenomena could naturally arise from backwarming which becomes strong when the flux maximum shifts into the UV region below $3000 \AA$.

\section{Non-DA Atmospheres}

It is evident from the spectra and especially from relative positions in the two-color diagrams that the atmospheric composition for the non-DA spectral types (DB, DC, $\lambda 4670$ ) is different. (Greenstein, 1958, 1960, Weidemann, 1968).

Knowing with certainty that the interior of degenerate stars must be hydrogen-free we may be surprised to find hydrogen in the atmospheres at all. The fact that we observe hydrogen spectra in the DA's does not imply that $\mathrm{H}$ is the most abundant atmospheric constituent. First estimates (Weidemann, 1963) and subsequent calculations by Nariai and Klinglesmith for pure H/He mixtures (1969) and for models with 
variable $\mathrm{H} / \mathrm{He}$ ratios including metals by Bues (1970) do show clearly that due to the enormous efficiency of $\mathrm{H}$ absorption the visual spectra of stars with $\log g=8$, $T_{\text {eff }}=15000 \mathrm{~K}$ remain hydrogen dominated even down to $\mathrm{H} / \mathrm{He}$ ratios of $10^{-3}$.

Vice versa Miss Bues was able to show that the complete absence of hydrogen lines in the HeI-line DB spectra forces the atmospheric hydrogen fraction down to about $10^{-5}$. This extremely low value was confirmed by a detailed study of the helium line profiles, whereas from colors alone an upper limit for $\mathrm{H} / \mathrm{He}$ of $10^{-4}$ was derived which is independent of metal content. The fact that in DB atmospheres hydrogen is completely absent is certainly of evolutionary significance. Since there is further indication that heavy elements are also underabundant (about a factor of 10 to 100 as compared with solar composition) we have to deal with atmospheres which consist of helium to a degree of purity never encountered in other astronomical objects.

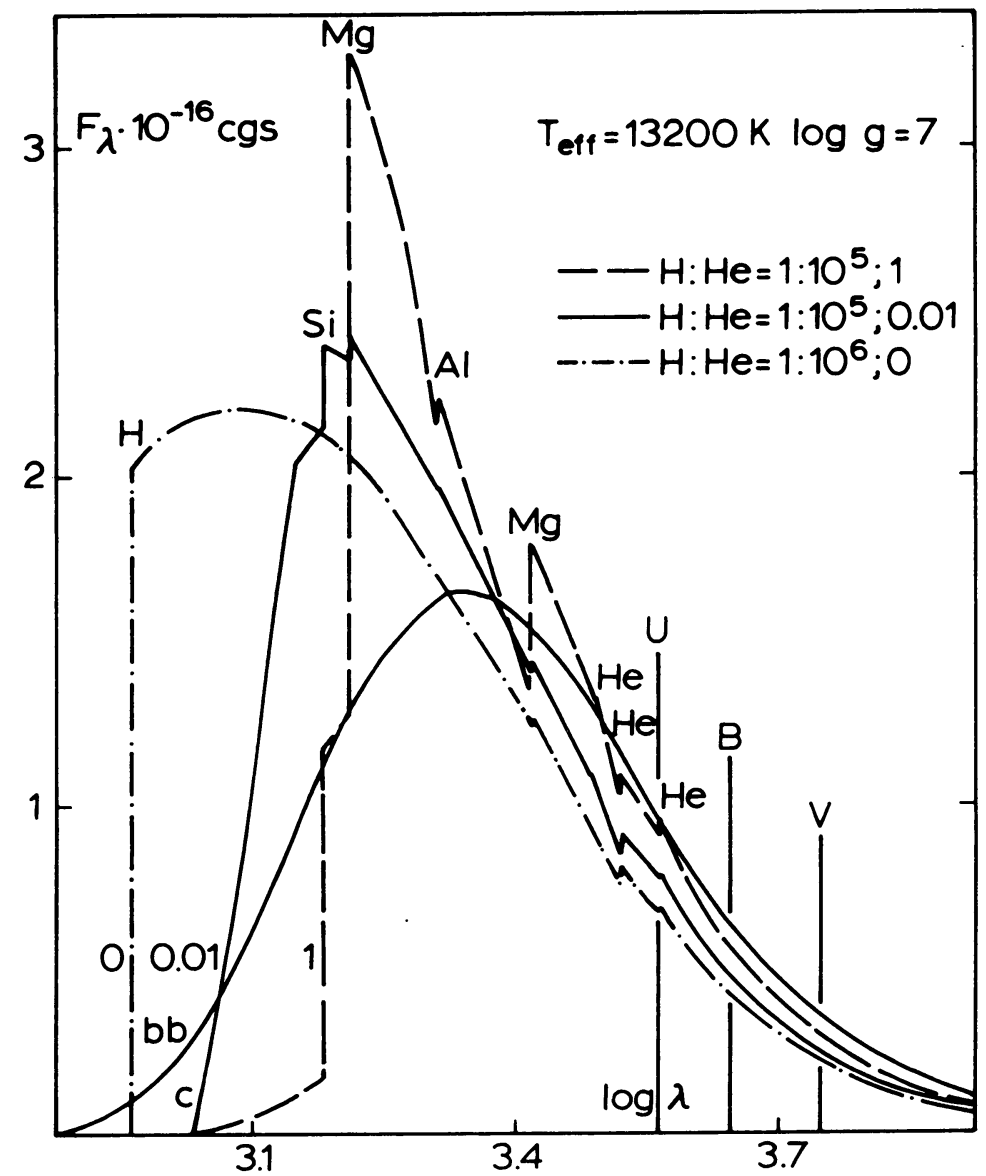

Fig. 11. Flux distribution for a DB model, according to Bues (1970), demonstrating the importance of metal absorption in the UV and its influence on the visual emergent flux ( $\mathrm{U}, \mathrm{B}$, and $\mathrm{V}$ marked). Different mixtures as indicated: 1: solar metal abundance, 0.01: $1 \%$ solar metal abundance. bb: black-body distribution. 
Although the metal content may be low it cannot be neglected in the construction of DB model atmospheres. Figure 11 demonstrates how the important absorption edges of $\mathrm{Mg}$, Si and $\mathrm{C}$ in the far UV cause the flux distribution in the visual region to steepen beyond that of the corresponding black body. This effect may in reality be even larger since the models do not include metal line blanketing which again - as in the case of the DA's - should increase the backwarming. This has immediate consequences for the temperature scale of the DB's: they do appear hotter than they actually are. Since for DB stars a Paschen like slope continues from the $\mathrm{V}$ via the $\mathrm{B}$ to the $\mathrm{U}$ band the shifting-vector from a black body to a line-free star in the two-color diagram should point along the black-body line to the upper left of Figure 3 - a fact which again has been confirmed by Miss Bues calculations. We thus understand her result that the DB stars are actually much cooler than anticipated from the UBV position relative to the DA's or black-bodies. Indeed the relative shift between a DA and a DB star of equal effective temperature $(12000 \mathrm{~K}, \log g=8)$ amounts to $\Delta(\mathrm{U}-\mathrm{V})=0.6$ (Figure 10). This demonstrates which extreme care should be taken in interpreting relative positions in color-magnitude diagrams as being due to relative changes in radii (or, if completely degenerate configurations are assumed, masses): the fact that most nonDA stars are located below the bulk of the DA's in the $M_{v}-(\mathrm{U}-\mathrm{V})$ diagram essentially reflects the absence of a Balmer discontinuity and does not give any information about differences in masses and radii between DA and non-DA white dwarfs. Since in the case of the comparatively rare DB's there are no close objects and no reliable distance determinations and since furthermore the spectra depend only little on gravity we are unfortunately not able to check if the $M-R$ relation is fulfilled. Yet it can again be stated that nothing is contradicted. (For a more detailed discussion of the DB stars I refer to Miss Bues' publication.) With regard to the atmospheric conditions we add that with $\log P_{g} \approx 7.5$ and $\log P_{e} \approx 4$ we have extremely neutral matter, with electrons provided by the metals and $\mathrm{He}^{-}$as the most important opacity source in the visual region.

The trend towards neutrality and higher densities continues with decreasing effective temperatures. Calculations for cooler model atmospheres are at present being made in view of a possible explanation of other non-DA white dwarfs which are located below the DB group in the two-color diagrams, more or less closely along the blackbody line. Whereas Miss Bues (1970) checked on the visibility of the H and K lines, Kumar and Doyle (1970) state that the CI white dwarf EG 182 and the $\lambda 4670$ stars are definitely rich in carbon. Since this is not the case for the DB's it seems that the minimum hypothesis of one second extremely hydrogen-deficient non-DA sequence of cooling white dwarfs (Weidemann, 1968) connecting DB, DC, $\lambda 4670$, and possibly DG stars cannot longer be upheld. On the other hand it might still be possible that cooling DB stars become DC objects. With regard to the DC stars which show only continuous spectra Wickramasinghe and Strittmatter (1970a) checked a suggestion by Ostriker and Bodenheimer (1968) that these might be normal white dwarfs for which extreme rotation has washed out the lines. Their calculations show that although this could be the case for absorption lines of other elements it cannot cause the hy- 
drogen lines to disappear. Investigations for extreme hydrogen-poor atmospheres at lower temperatures become hampered by an increased tendency to molecule or quasimolecule formation which changes both the equilibrium composition and the opacity sources.

The fact that molecule formation plays an important role at solar temperatures had already been demonstrated in the analysis of the atmosphere of van Maanen 2, the only representative of spectral type DG (Weidemann, 1960). In this case it was found that for the most probable model with $\log g=8$ and solar effective temperature hydrogen atoms and molecules should be present in equal amounts, whereas the total hydrogen abundance compared to helium had to be reduced at least ten times in order to yield the high transparency necessary for the explanation of the extreme pressure broadening of the observed iron lines. Another unexpected result was the conclusion that metals should be underabundant by a factor of 10000 , slightly dependent on the assumed temperature. The atmospheric pressure was estimated to be around $200 \mathrm{~atm}$ $\left(\log P_{g}=9.3\right)$. From recent observations in the infrared (Eggen, 1970) and from Strömgren colors (Graham, 1969) we now find that van Maanen 2 should be slightly hotter than the sun with $T_{\text {eff }} \approx 6700 \mathrm{~K}$ and a corresponding reduction of molecule formation. However even in this case the atmosphere remains extremely neutral with $\log \left(P_{g} / P_{e}\right)$ about $10^{7}$. With a smaller radius $\log g$ increases to about 8.5 and the mass to $0.9 M_{\odot}$ if the mass-radius relation is assumed to hold. Under such conditions the scale height of the atmosphere shrinks to $4 \mathrm{~m}$ only (as compared to $200 \mathrm{~km}$ in the sun). Is is evident that care must be taken to apply the usual theory, f.e. of pressure broadening. The fact that the Fe lines in vMa 2 are symmetrically broadened on the other hand gives an independent hint that helium with its low polarizibility is the main broadening agent.

Studies on the possibility of convection in the outer nondegenerate envelopes of hydrogen-poor white dwarfs have been carried out by Böhm $(1968,1969)$ van Horn (1970) and Grenfell and Böhm (1970). Although these papers aim mainly at the question of energy transport from the interior to the surface (in connection with the cooling theory) it is interesting in our context to note that the upper boundary of a $\mathrm{He}^{-}$ convection zone for a set of models with van Maanen 2's composition and effective temperatures between 5000 and $14000 \mathrm{~K}$ (with absorption of $\mathrm{H}, \mathrm{H}^{-}, \mathrm{He}^{-}, \mathrm{C}, \mathrm{N}, \mathrm{O}$, $\mathrm{C}^{-}, \mathrm{O}^{-}, \mathrm{Mg} \mathrm{I}$ and $\mathrm{Si}$, as well as $\mathrm{H}_{2}$-molecule formation included) reaches its maximum for $T_{\text {eff }}$ about $6000 \mathrm{~K}$ at an optical depth of about 0.5 . As in the case of DA and hot pure-helium atmospheres which have been studied by Wickramasinghe and Strittmatter (1970b) convection turns out to be practically unimportant for the interpretation of the visual spectra: whereas the equivalent widths of $\mathrm{H} \gamma$ and $\mathrm{He}$ I $\lambda 4472$ are increased by about $10 \%$ - well below observational and theoretical uncertainties - the colors do even change less $(0.01 \mathrm{mag}$ in $\mathrm{U}-\mathrm{B}$ and $\mathrm{B}-\mathrm{V})$. This insensitivity of colors and equivalent widths to slight changes of temperature stratification has also been demonstrated for DA atmospheres with varying $\mathrm{H} / \mathrm{He}$ ratios by Matsushima and Terashita (1969b).

It thus appears that in the construction of real white dwarf atmospheres convection 
is less important than the inclusion of other opacity sources and the consideration of blanketing effects for all ranges of temperatures and composition. In view of the scarcity of information it is hoped that new observations in a broader spectral range (e.g. multichannel data) might help us to narrow down the range of possibilities with which we certainly will have to live for some time to come.

\section{References}

Adams, T. F. and Morton, D. C.: 1968, Astrophys. J. 152, 195.

Bues, I.: 1970, Astron. Astrophys. 7, 91.

Böhm, K. H.: 1968, Astrophys. Space Sci. 2, 375.

Böhm, K. H.: 1969, in Low Luminosity Stars (ed. by S. S. Kumar), Gordon and Breach, New York, p. 393.

Davis, J. and Webb, R. J.: 1970, Astrophys. J. 159, 551.

Eggen, O.: 1969, Astrophys. J. 157, 287.

Eggen, O.: 1970, Astrophys. J. 159, 945.

Eggen, O. and Greenstein, J. L.: 1967, Astrophys. J. 150, 927.

Greenstein, J. L.: 1958, in Encyclopedia of Physics 50, Springer-Verlag, Berlin, p. 161.

Greenstein, J. L.: 1960, in Stars and Stellar Systems 6 (ed. by J. L. Greenstein), University Chicago Press, Chicago, p. 676.

Greenstein, J. L.: 1969, Astrophys. J. 158, 281.

Graham, J. A.: 1969, in Low Luminosity Stars (ed. by S. S. Kumar), Gordon and Breach, New York, p. 139.

Graham, J. A.: 1970, White Dwarf Photometry: Extended List, private communication.

Grenfell, T. C. and Böhm, K. H.: 1970, Astrophys. J. 161,1183.

Hamada, T. and Salpeter, E. E.: 1961, Astrophys. J. 134, 683.

Luyten, W. J.: 1967, Publ. Astron. Obs. Minnesota 3, Nr. 19.

Matsushima, S.: 1969, Astrophys. J. 158, 1137.

Matsushima, S. and Terashita, Y.: 1969a, in Low Luminosity Stars (ed. by S. S. Kumar), Gordon and Breach, New York, p. 315.

Matsushima, S. and Terashita, Y.: 1969b, Astrophys. J. 156, 183.

Matsushima, S. and Terashita, Y.: 1969c, Astrophys. J. 156, 219.

Nariai, K. and Klinglesmith, D. A.: 1969, in Theory and Observation of Normal Stellar Atmospheres, Proc. 3rd Harvard Conf. (ed. by O. Gingerich), MIT Press, Cambridge, Mass. and London, p. 315.

Ostriker, J. P. and Bodenheimer, P.: 1968, Astrophys. J. 151, 1089.

Terashita, Y. and Matsushima, S.: 1966, Astrophys. J. Suppl. 13, 461.

Terashita, Y. and Matsushima, S.: 1969, Astrophys. J. 156, 203.

Unsöld, A.: 1955, in Physik der Sternatmosphären, 2nd ed., Springer-Verlag, Berlin, p. 202ff and p. 479.

Van Altena, W. F.: 1966, Publ. Astron. Soc. Pacific 78, 345.

Van Horn, H. M.: 1970, Astrophys. J. 160, L53.

Vitense, E.: 1951, Z. Astrophys. 29, 73.

Weidemann, V.: 1960, Astrophys. J. 131, 638.

Weidemann, V.: 1963, Z. Astrophys. 57, 87.

Weidemann, V.: 1966, J. Q. Spectr. Radiative Transfer 6, 691.

Weidemann, V.: 1968, Ann. Rev. Astron. Astrophys. 6, 351.

Wickramasinghe, D. T. and Strittmatter, P. A.: 1970a, Monthly Notices Roy. Astron. Soc. $147,123$.

Wickramasinghe, D. T. and Strittmatter, P. A.: 1970b, Monthly Notices Roy. Astron. Soc. 150, 435. 